City University of New York (CUNY) CUNY Academic Works

1982

\title{
Aspects of duality in chiral field theories
}

\author{
A. P. Balachandran \\ Syracuse University \\ V. Parameswaran Nair \\ City College of New York \\ B. S. Skagerstam \\ Centre Européen de Recherches Nucléaires \\ C. G. Trahern \\ Syracuse University
}

\section{How does access to this work benefit you? Let us know!}

More information about this work at: https://academicworks.cuny.edu/cc_pubs/380

Discover additional works at: https://academicworks.cuny.edu

This work is made publicly available by the City University of New York (CUNY).

Contact: AcademicWorks@cuny.edu 


\title{
Aspects of duality in chiral field theories
}

\author{
A. P. Balachandran and V. P. Nair \\ Physics Department, Syracuse University, Syracuse, New York 13210 \\ B. S. Skagerstam* \\ Centre Européen de Recherches Nucléaires, Geneva, Switzerland \\ C. G. Trahern \\ Physics Department, Syracuse University, Syracuse, New York 13210 \\ (Received 31 July 1981)
}

\begin{abstract}
The Abelian and non-Abelian gauge potentials $A_{\mu \nu}$ are known to describe chiral fields. Here we study their interactions with dual extended objects. The region occupied by these objects is shown to behave like a distinct phase of chiral fields which is nondissipative under suitable geometric conditions. A method for the realization of the ' $t$ Hooft algebra in these systems is outlined. The concept of electric and magnetic duality in electromagnetism is generalized to chiral fields with values in a symmetric space.
\end{abstract}

\section{INTRODUCTION}

It has been known for some time that phonon modes (massless scalar fields) in theories with an Abelian group of symmetries can be described by antisymmetric potentials $A_{\mu \nu} \cdot{ }^{1,2}$ Such a description is interesting on at least two counts: (a) it is well adapted to the description of Abrikosov ${ }^{3}$ (or Nielsen-Olesen ${ }^{4}$ ) vortex lines; (b) it is invariant under the gauge transformation

$$
A_{\mu \nu} \rightarrow A_{\mu \nu}+\partial_{\mu} \Lambda_{\nu}-\partial_{\nu} \Lambda_{\mu}
$$

which generalizes the electromagnetic gauge transformation

$$
A_{\mu} \rightarrow A_{\mu}+\partial_{\mu} \Lambda \text {. }
$$

It is thus a new sort of gauge theory.

Conventional phonon (or massless scalar) fields are examples of "chiral" or "nonlinear" fields in an Abelian theory. Deser ${ }^{1}$ and Freedman and Townsend ${ }^{5}$ have also formulated non-Abelian nonlinear models in terms of (Lie-algebra valued) antisymmetric potentials $A_{\mu \nu}$. They describe interacting non-Abelian massless scalar fields. In this paper, we study the interaction of dual extended objects with such $A_{\mu \nu}$. This paper is a generalization of an earlier work on extended objects in interaction with Abelian $A_{\mu \nu}{ }^{6}$

Such an investigation seems worthwhile for several reasons.

(A) As is well known, the chiral-symmetry phase is a broken-symmetry phase. Regions of vortices and their condensates (like strings, shells, and bags) on the other hand describe a distinct phase where symmetry is restored. The existence of this symmetric phase is of interest in the study of chiral field theories (for example at finite temperatures). Furthermore, these domains of higher symmetry have features in common with the MIT bag. ${ }^{7}$

(B) These strings and extended objects are described by dual Lagrangians ${ }^{8}$ modified by interaction terms with chiral fields. Both dual extended objects and chiral fields seem to describe aspects of reality. We are therefore encouraged to study their mutual interaction.

(C) We show that chiral models with vortices lead to a realization of the ' $t$ Hooft commutation relations. ${ }^{9}$ There is reason to believe that an understanding of this algebraic structure may clarify the phases of quantum chromodynamics (QCD).

(D) Free electromagnetism admits mutually dual descriptions: it can be described by introducing a potential $A_{\mu}$ either for the field strength $F_{\mu \nu}$ or a dual potential $B_{\mu}$ for the dual field strength $* F_{\mu \nu}$. The description of phonon modes by Lie-algebra-valued fields is dual in a similar sense. ${ }^{10}$ These dual descriptions merit attention in view of the rich topological features and physical results (like the Dirac quantization condition ${ }^{11}$ and the Feynman-Onsager relation ${ }^{10}$ ) they lead to.

The contents of this paper are as follows. Section II recalls earlier work on the Abelian gauge potentials $A_{\mu_{\nu}}$ and their interaction with extended objects. We emphasize that due to reparametrization invariance, the regions occupied by the extended objects behave rather like a different phase of phonons which under suitable geometrical conditions in nondissipative and hence stable (these systems have similarities to a superconductor in an ambient electromagnetic field). Section III begins with a review of earlier work on non-Abelian $A_{\mu \nu}$. The proof of the equivalence of these models to those nonlinear models where fields have values in a (semisimple, compact Lie) group $G$ is in- 
dicated. It is also slightly generalized to describe nonlinear models where fields have values in a homogeneous space $G / H$ (where $H$ is a subgroup of $G$ ). We then discuss the gauge-invariant interactions of non-Abelian $A_{\mu \nu}$ to strings, shells, and bags. As in the Abelian case, ${ }^{6}$ properties similar to superconductivity (or vortices in a superfluid) are found as a consequence of reparametrization invariance. Section IV begins with a general discussion of the manner in which vortices can arise in a Higgs field. We then remark on a suggestive resemblance between the Deser-Freedman-Townsend (D FT) Lagrangian and a Yang-Mills Lagrangian with Higgs fields (see also Sugamoto, and Seo et al. ${ }^{1}$ ). We also argue that the latter can exhibit vortex production; a domain where these vortices condense then constitutes a domain where symmetry is restored. Section V presents an analysis of the DFT system and the ' $t$ Hooft algebra. It is shown that the dynamical variables of the DFT Lagrangian with vortex sources can be used to realize 't Hooft's algebra of $A$ and $B$ operators. Finally, in Sec. VI, we discuss the concept of duality for the new gauge systems. It is found that while the concept is formulated with ease if such systems are Abelian, it can be formulated for the non-Abelian case only if the space $G / H$ of chiral fields is a symmetric space. ${ }^{12}$

\section{THE ABELIAN $\boldsymbol{A}_{\mu \nu}$}

In this section, we briefly recall earlier work on the interactions of $A_{\mu_{\nu}}$ to extended objects.

The potential $A_{\mu_{\nu}}$ is antisymmetric in $\mu$ and $\nu$ and is subject to the gauge transformations

$$
A_{\mu_{\nu}} \rightarrow A_{\mu_{\nu}}+\partial_{\mu} \Lambda_{\nu}-\partial_{\nu} \Lambda_{\mu}
$$

The associated gauge-invariant field strength is

$$
F_{\mu \nu \lambda}=\partial_{\mu} A_{\nu \lambda}+\partial_{\nu} A_{\lambda \mu}+\partial_{\lambda} A_{\mu \nu} .
$$

In analogy to Lorentz and Maxwell equations, we now postulate equations for the interactions of extended objects and $F_{\mu_{\nu \lambda}}$. Let

$$
z(\sigma) \equiv z\left(\sigma^{0}, \sigma^{1}, \ldots, \sigma^{n-1}\right), \quad n \geqslant 2
$$

describe the extended object $E$ in Minkowski space and let $\mathscr{L}_{0}$ be the Nambu-Goto Lagrangian density. ${ }^{8}$ Then we write

$$
\begin{aligned}
& \partial_{a} \frac{\partial \mathscr{L}_{0}}{\partial\left(\partial_{a} z^{\mu}\right)}=-\frac{1}{2} F_{\mu \nu \lambda}(z) \sigma^{\nu \lambda}, \\
& \partial_{\mu} F^{\mu \nu \lambda}(x)=\int_{E} d^{n} \sigma \delta^{4}(x-z(\sigma)) \sigma^{\nu \lambda}(\sigma) .
\end{aligned}
$$

Here

$$
\begin{aligned}
& \partial_{a} \equiv \frac{\partial}{\partial \sigma^{a}}, \\
& \sigma^{\nu \lambda}=J^{a b} \partial_{a} z^{\nu} \partial_{b} z^{\lambda}, \\
& J^{a b}=-J^{b a} .
\end{aligned}
$$

(We use the conventions $a_{\mu} b^{\mu}=\overrightarrow{\mathrm{a}} \cdot \overrightarrow{\mathrm{b}}-a_{0} b_{0}, \epsilon_{0123}$ $=-\epsilon^{0123}=1$.) We interpret $J^{a_{b}}$ as the analog of a current on $E$; it is a measure of the coupling between $E$ and $F_{\mu \nu \lambda}$. For a string,

$$
\begin{aligned}
& J^{a b}=\omega \epsilon^{a b}, \\
& \epsilon^{10}=1 .
\end{aligned}
$$

When $J^{a b}=0, F_{\mu \nu \lambda}$ describes a massless scalar field. For then, if we write

$$
F_{\mu \nu \lambda}=\epsilon_{\mu \nu \lambda \rho} M^{\rho}
$$

Eq. (2.5) gives

$$
\partial_{\mu} M_{\nu}-\partial_{\nu} M_{\mu}=0
$$

so that

$$
M_{\mu}=\partial_{\mu} \varphi
$$

for some $\varphi$. The Bianchi identity

$$
\epsilon^{\mu \nu \lambda \rho} \partial_{\mu} F_{\nu \lambda \rho} \equiv 0
$$

then shows that $\varphi$ is a massless scalar field

$$
\partial^{2} \varphi=\partial_{\mu} M^{\mu}=0 .
$$

Let us now consider the case $J^{a b} \neq 0$. The identity

$$
\partial_{\nu} \partial_{\mu} F^{\mu \nu \lambda} \equiv 0
$$

then gives the conservation law

$$
\partial_{a} J^{a b}=0,
$$

which, for $n=2$, says that $\omega$ is a constant. The identity

$$
\partial_{b} z^{\mu} \partial_{a} \frac{\partial \mathcal{L}_{0}}{\partial\left(\partial_{a} z^{\mu}\right)} \equiv 0
$$

[which is due to the reparametrization invariance of $\mathscr{L}_{0}$ (cf. Ref. 13)] gives

$$
F_{\mu \nu \lambda}(z) \partial_{b} z^{\mu} \sigma^{\nu \lambda}=0 \text {. }
$$

This identity has the following remarkable consequences ${ }^{13,6}$ : (a) If the extended body is static, that is if $z(\sigma)$ is time independent, it does not dissipate energy even if $J^{a b} \neq 0$; this behavior resembles nondissipation of energy by a superconductor; (b) the extended object tends to be impenetrable to the "velocity" field $M_{\mu}$. This behavior resembles the Meissner effect.

The following Lagrangian density for this system when $J^{a b}=0$ is due to Deser ${ }^{1}$ and Freedman and Townsend ${ }^{5}$ :

$$
\mathscr{L}_{F}=\frac{f}{4} e^{\mu \nu \lambda \rho} F_{\mu \nu}(P) A_{\lambda \rho}-\frac{f^{2}}{2} P_{\mu} P^{\mu},
$$




$$
F_{\mu \nu}(P) \equiv \partial_{\mu} P_{\nu}-\partial_{\nu} P_{\mu}
$$

(here, $f$ is a constant). For, variation of $P_{\mu}$ shows that

$$
f P_{\mu}=\frac{1}{3 !} \epsilon_{\mu \nu \lambda \rho} F^{\nu \lambda \rho} .
$$

Substitution in $\mathscr{L}_{F}$ reduces it to the standard form ${ }^{5}$

$$
\mathscr{L}_{F}=-\frac{1}{12} F_{\mu \nu \lambda} F^{\mu \nu \lambda} \text {. }
$$

Interaction of $F_{\mu \nu \lambda}$ with $E$ can be described by the action $^{6}$

$$
\begin{aligned}
& S_{I}=\int_{E} d^{n} \sigma \mathcal{L}_{I}, \\
& \mathcal{L}_{I}=-\frac{1}{2}\left[A_{\mu \nu} \sigma^{\mu \nu}-J^{a b}\left(\partial_{a} x_{b}-\partial_{b} x_{a}\right)\right] .
\end{aligned}
$$

Full variation of $\omega$ leads to the stronger result

$$
F_{\mu \nu \lambda} \partial_{a} z^{\mu} \partial_{b} z^{\nu} \partial_{c} z^{\lambda}=0
$$

rather than to (2.19). Modifications required to obtain (2.19) are discussed in Ref. 6.

\section{THE NON-ABELIAN $\boldsymbol{A}_{\mu \nu}$}

We consider now the case where $A_{\mu \nu}$ and $P_{\mu}$ are Lie-algebra valued. Let $G$ be the internal-symmetry group, $G$ its Lie algebra, and

$$
L(\alpha), \alpha=1,2, \ldots,[G]
$$

a (Hermitian) basis for $G$. Here $[G]$ is the dimension of $G$, and $L(\alpha)$ are normalized according to

$$
\operatorname{Tr} L(\alpha) L(\beta)=\delta_{\alpha_{\beta}} \text {. }
$$

[We assume that $G, G$, and $L(\alpha)$ are given concretely in terms of matrices. 1 Let

$$
\begin{aligned}
& A_{\mu \nu}=i A_{\mu \nu}^{\alpha} L(\alpha), \\
& P_{\mu}=i P_{\mu}^{\alpha} L(\alpha), \\
& F_{\mu \nu}(P)=\partial_{\mu} \dot{P}_{\nu}-\partial_{\nu} P_{\mu}+\left[P_{\mu}, P_{\nu}\right]
\end{aligned}
$$

[the fields $A_{\mu \nu}, P_{\mu}$, and $F_{\mu_{\nu}}$ are anti-Hermitian unlike the ones in (2.20)]. The Deser-FreedmanTownsend generalization of $(2.20)$ is

$$
\mathscr{L}_{F}=-\frac{f}{4} \epsilon^{\mu \nu \lambda \rho} \operatorname{Tr} F_{\mu \nu}(P) A_{\lambda \rho}+\frac{f^{2}}{2} \operatorname{Tr} P_{\mu} P^{\mu} .
$$

This Lagrangian density describes group-valued chiral fields. For, variation of $A_{\lambda \rho}$ shows that

$$
F_{\mu \nu}(P)=0
$$

or

$$
P_{\mu}=g^{-1} \partial_{\mu} g
$$

for some $g(x) \in G$. Substitution in $\mathscr{L}_{F}$ reduces it to the nonlinear Lagrangian

$$
\frac{f^{2}}{2} \operatorname{Tr}\left(g^{-1} \partial_{\mu} g\right)\left(g^{-1} \partial^{\mu} g\right) \text {. }
$$

Note that $\mathscr{L}_{F}$ is invariant (up to surface terms) under the gauge transformation

$$
\begin{aligned}
& A_{\mu \nu} \rightarrow A_{\mu \nu}+D_{\mu}(P) \Lambda_{\nu}-D_{\nu}(P) \Lambda_{\mu}, \\
& D_{\mu}(P) \Lambda_{\nu} \equiv \partial_{\mu} \Lambda_{\nu}+\left[P_{\mu}, \Lambda_{\nu}\right], \\
& \Lambda_{\nu}(x) \in \underline{G} .
\end{aligned}
$$

As a consequence of the Bianchi identity

$$
\epsilon^{\mu \nu \lambda \rho} D_{\nu}(P) F_{\lambda \rho}(P)=0,
$$

such a transformation is analogous to (2.1).

$$
\text { Generalizations of (3.6) }
$$

The general nonlinear model is defined on a homogeneous space $G / H$ where $H$ is a subgroup of $G$. We can describe these as well by modifying (3.6) as follows (see Ref. 14 for an alternative approach).

Let the Lie algebra $\underline{H}$ of $H$ be spanned by $T(\alpha)$ :

$$
L(\alpha)=T(\alpha), \quad \alpha=1,2, \ldots, \text { dimension of } H=[H] .
$$

Let the orthogonal complement of $\underline{H}$ be spanned by $S(i)$ :

$$
\begin{aligned}
& L([H]+i)=S(i), \quad i=1,2, \ldots,[G]-[H], \\
& \operatorname{Tr} S(i) T(\alpha)=0 .
\end{aligned}
$$

Then (3.6) is replaced by

$$
\begin{aligned}
\mathscr{L} & =\frac{f^{2}}{2} \operatorname{Tr} \hat{P}^{2}-\frac{f}{4} \epsilon^{\mu \nu \lambda \rho} \operatorname{Tr}\left(F_{\mu \nu} A_{\lambda \rho}\right), \\
\hat{P}_{\mu} & =S(i) \operatorname{Tr} S(i) P_{\mu} \\
& \equiv S(i) \hat{P}_{\mu}^{i} .
\end{aligned}
$$

Since (3.7) and (3.8) are still true, substitution in (3.18) leads to

$$
\mathcal{L}=\frac{f^{2}}{2} \operatorname{Tr}\left[S(i) g^{-1} \partial_{\mu} g\right] \operatorname{Tr}\left[S(i) g^{-1} \partial^{\mu} g\right] .
$$

We know from Ref. 15 that this describes nonlinear models on $G / H$.

There is a further generalization of (3.6) and (3.20) which will be useful in Sec. IV. First note that the reason why $(3.20)$ describes nonlinear models on $G / H$ is the following. If $h(x) \in H$,

$$
\operatorname{Tr} S(i)(g h)^{-1} \partial_{\mu}(g h)=\operatorname{Tr}\left[h S(i) h^{-1}\right] g^{-1} \partial_{\mu} g
$$

since $h^{-1} \partial_{\mu} h \in \underline{H}$ and

$$
\operatorname{Tr} S(i) h^{-1} \partial_{\mu} h=0
$$

by (3.16). Further,

$$
h S(i) h^{-1}=\gamma(h)_{j i} S(j),
$$

where $\{\gamma(h)\}$ is some real orthogonal representation of $H$. (To prove this, note the following: (a) 
$h^{-1} T(\alpha) h \in H$ and therefore $\operatorname{Tr} T(\alpha) h S(i) h^{-1}$

$=\operatorname{Tr} h^{-1} T(\alpha) \bar{h} S(i)=0$; thus there are no $T(\alpha)$ terms on the right-hand side of (3.23); (b) $\gamma(h)$ is real since $S(i)$ and $h S(i) h^{-1}$ are Hermitian; (c) $\gamma(h)$ is orthogonal since $\operatorname{Tr}\left[h S(i) h^{-1}\right]\left[h S(j) h^{-1}\right]=\operatorname{Tr} S(i) S(j)$. Thus $\mathscr{L}$ is gauge invariant under $g \rightarrow g h$. It follows that the physical fields are not $g$, but rather fields with values in $G / H=\left\{g H \equiv g\left\{h_{i}\right\}_{h_{i} \in H}\right\}$.)

If $\{\gamma(h)\}$ is reducible, there are in general many matrices $m=\left(m_{i j}\right)$ with the properties

$$
\gamma(h) m \tilde{\gamma}(h)=m, \quad m \neq \text { multiple of identity . }
$$

Then (3.20) can be replaced by

$$
\frac{f^{2}}{2} m_{i j} \operatorname{Tr} S(i) g^{-1} \partial_{\mu} g \operatorname{Tr} S(j) g^{-1} \partial^{\mu} g
$$

without spoiling gauge invariance under $H$. Thus we can generalize $(3.17)$ to

$$
\begin{gathered}
\mathcal{L}=-\frac{f}{4} \epsilon^{\mu \nu \lambda \rho} \operatorname{Tr} A_{\mu \nu} F_{\lambda \rho}(P)+\frac{f^{2}}{2} m_{i j} \hat{P}_{\mu}^{i} \hat{P}^{\mu j} . \\
\text { Interaction with strings }
\end{gathered}
$$

A simple modification of (2.23) and (2.24) can describe the interaction of non-Abelian $A_{\mu_{\nu}}$ with extended objects. We write

$$
\begin{aligned}
& S_{I}=\int_{E} d^{n} \sigma \mathcal{L}_{I}, \\
& \mathcal{L}_{I}=+\frac{i}{2}\left\{\operatorname{Tr} A_{\mu \nu} \sigma^{\mu \nu}-\operatorname{Tr} J^{a b}\left[D_{a}(P) \chi_{b}-D_{b}(P) \chi_{a}\right]\right\},
\end{aligned}
$$

where

$$
\begin{aligned}
& \sigma^{\mu \nu}=J^{a b} \partial_{a} z^{\mu} \partial_{b} z^{\nu}, \\
& J^{a b}=-J^{b a} .
\end{aligned}
$$

Here $J^{a b}$ and $i \chi^{a}$ are Lie-algebra valued and Hermitian, and, for a string,

$$
J^{a b}=\epsilon^{a b} \omega .
$$

Note that $\mathscr{L}_{I}$ is invariant under (3.10) if we also change $\chi_{a}$ according to

$$
\chi_{a} \rightarrow \chi_{a}+\Lambda_{\mu} \partial_{a} z^{\mu} \text {. }
$$

In discussing the equations of motion for this $\mathscr{L}_{I}$, we will vary $\omega$ fully. This will lead to the stronger equation (2.25) rather than to (the trace of) (2.19). (The latter can be obtained if $J^{a b}$ is varied only by reparametrizations, cf. Ref. 6.) Furthermore, we will only consider the case $H=\{$ identity $\}$ so that $\mathcal{L}_{F}$ is of the form (3.6).

We want to show that the variational problem leads to the following generalization of the Abelian equations:

(1) $f F_{\mu \nu}(P)+\frac{i}{2} \int_{E} d^{2} \sigma \delta^{4}(x-z(\sigma)) \epsilon_{\mu \nu \rho \lambda} \sigma^{\rho \lambda}(\sigma)=0$
(2) The field $P$ provides a source term for the string:

$$
\partial_{a} \frac{\partial \mathcal{L}_{0}}{\partial\left(\partial_{a} z^{\mu}\right)}=i \frac{f}{2} \epsilon_{\mu \alpha \beta \rho} \operatorname{Tr} P^{\rho} \sigma^{\alpha \beta}
$$

(3) The field $P$ is conserved:

$D_{\mu}(P) P^{\mu}=\partial_{\mu} P^{\mu}=0$.

(4) There is current conservation:

$$
D_{a}(P) \omega=0 \text {. }
$$

The following remarks can be made: (a) (3) is fulfilled as an identity in the Abelian case due to its expression in terms of $A_{\mu_{\nu}}$; (b) the Bianchi identity on $F_{\mu \nu}(P)$ leads from (3.33) to (3.36) and (4) also follows by varying $\chi_{a}$ in (3.8); (c) as usual the consequence of the identity $(2.18)$ is trivial for the string. We now derive (1), (2), and (3) above.

Variation of $A_{\mu_{\nu}}$ leads to (3.33). It implies in particular that on the string,

$$
\left[F_{\mu \nu}, \omega\right]=0=\left[F_{\mu_{\nu}}, \sigma_{\lambda \rho}\right] \text {. }
$$

Variation of $P_{\mu}$ gives

$f^{2} \epsilon_{\mu \nu \lambda \rho} P^{\rho}=f F_{\mu \nu \lambda}+i \epsilon_{\mu \nu \lambda \rho} \int_{E} d^{2} \sigma \delta^{4}(x-z) \epsilon^{a b} \partial_{a} z^{\rho}\left[\chi_{b}, \omega\right]$.

where

$$
F_{\mu \nu \lambda} \equiv D_{\mu}(P) A_{\nu \lambda}+D_{\nu}(P) A_{\lambda \mu}+D_{\lambda}(P) A_{\mu \nu} .
$$

Thus from (3.38)

$$
f \epsilon_{\mu \nu \lambda \rho} P^{\rho} \partial_{a} z^{\nu} \partial_{b} z^{\lambda}=F_{\mu \nu \lambda} \partial_{a} z^{\nu} \partial_{b} z^{\lambda} .
$$

Variation of $\omega$ gives

$$
\epsilon^{a b} A_{\mu \nu}(z) \partial_{a} z^{\mu} \partial_{b} z^{\nu}=\epsilon^{a b}\left[D_{a}(P) \chi_{b}-D_{b}(P) \chi_{a}\right] .
$$

Variation of $z^{\mu}$ in conjunction with the previous results leads to (3.34). Finally, we can compute $D_{\mu}(P) P^{\mu}$ from (3.38) and use (3.41), (3.33), and (3.36) to find (3.35).

Thus the system describes non-Abelian vortices in the sourceless $\underline{G}$-valued vector field $P^{\mu}$.

\section{Shells}

The analogs for Eqs. (3.33)-(3.36) are

$f F_{\mu_{\nu}}(P)+\frac{i}{2} \int_{E} d^{3} \sigma \delta^{4}(x-z(\sigma)) \epsilon_{\mu \nu \rho \sigma} \epsilon^{a b c} \omega_{a} \partial_{b} z^{\nu} \partial_{c} z^{\sigma}=0$,

$\partial_{a} \frac{\partial \mathcal{L}_{0}}{\partial\left(\partial_{a} z^{\mu}\right)}=i \frac{f}{2} \epsilon_{\mu_{\nu \alpha \beta}} \operatorname{Tr}\left(P^{\beta} \omega_{c}\right) \epsilon^{a b c} \partial_{a} z^{\nu} \partial_{b} z^{\alpha}$,

$\partial_{\mu} P^{\mu}=0$,

$\epsilon^{a b c} D_{b}(P) \omega_{c}=0$. 
The derivation of these equations from (3.27) (with $n=3$ ) follows the same steps as in the case of the string. The identity (2.18) implies

$$
\epsilon^{a b c} \epsilon_{\mu \nu \alpha \beta} \operatorname{Tr}\left(P^{\beta}(z) \omega_{c}\right) \partial_{a} z^{\nu} \partial_{b} z^{\beta} \partial_{d} z^{\mu}=0 .
$$

Unlike in the case of the string, this condition is not trivial. In analogy with the Abelian case [Eqs. (2.19) and (2.25)], on any portion of the shell which couples to the field, those components of $P_{\mu}$ which lie in the direction of $J^{a b}=\epsilon^{a b c} \omega_{c}$ (in the internal space) are tangential to the shell. Unlike the Abelian case, condition (3.46) does not restrict $P_{\mu}$ to be completely tangential. Weaker conditions of this type were also found for the interaction of Yang-Mills fields with shells. ${ }^{13}$

\section{Bags}

The interaction Lagrangian for the bag is

$$
\begin{aligned}
& \mathscr{L}_{I}= \frac{i}{2} \int_{E} d^{4} \sigma \delta^{4}(x-z(\sigma)) \operatorname{Tr} \\
& \times\left[A_{\mu \nu} \sigma^{\mu \nu}-J^{a b}\left(D_{a} \chi_{b}-D_{b} \chi_{a}\right)\right], \\
& \sigma^{\mu \nu}=\epsilon^{a b c d} \omega_{c d} \partial_{a} z^{\mu} \partial_{b} z^{\nu} .
\end{aligned}
$$

The equations of motion for this case are

$$
\begin{aligned}
& f F_{\mu \nu}+\frac{i}{2} \int_{E} d^{4} \sigma \delta^{4}(x-z(\sigma)) \epsilon_{\mu \nu \sigma \rho} \epsilon^{a b c d} \omega_{a b} \partial_{c} z^{\rho} \partial_{d} z^{\sigma}=0, \\
& \partial_{a} \frac{\partial \mathscr{L}_{0}}{\partial\left(\partial_{a} z^{\mu}\right)}=i \frac{f}{2} \operatorname{Tr}\left(P^{\beta} \omega_{c d}\right) \epsilon_{\mu \nu \alpha \beta} \epsilon^{a b c d} \partial_{a} z^{\nu} \partial_{b} z^{\alpha}, \\
& \partial_{\mu} P^{\mu}=0, \\
& \epsilon^{a b d} D_{b}(P) \omega_{c d}=0 .
\end{aligned}
$$

One can now follow the arguments used in the Abelian case to show the following. In the interior of the bag, Eqs. (3.47) and (3.48) imply

$$
f F_{\mu \nu}(P)=-\frac{i}{2} \epsilon_{\mu \nu \alpha \beta} \frac{\partial(\sigma)}{\partial(z)} J^{a b} \partial_{a} z^{\alpha} \partial_{b} z^{\beta}
$$

and

$$
\epsilon_{\mu \nu \alpha \beta} \operatorname{Tr}\left(P^{\beta} J^{a b}\right) \partial_{a} z^{\nu} \partial_{b} z^{\alpha}=0 .
$$

where $J^{a b}=\epsilon^{a b c d} \omega_{c d}$. Equation (3.53) follows from (3.49) because the left-hand side vanishes in the interior of the bag [see (6.20) in Sec. VI]. Combining Eq. (3.53) with (3.52) leads to

$$
\operatorname{Tr}\left(P^{\mu} F_{\mu \nu}\right)=0
$$

in the interior of the bag.

We notice that (3.53) tends to exclude $P_{\mu}$ from the interior of the bag. If the bag has a surface, there are equations like (3.46) on the surface with consequences which have been indicated.
As in previous work ${ }^{6}$ nondissipation theorems can be proved for extended objects in interaction with non-Abelian $A_{\mu \nu}$. They are due to the identity (2.18) and its consequences like (3.46) and (3.55).

\section{VORTICES, HIGGS FIELDS, AND GENERALIZED GAUGE FIELDS}

As explained in the Introduction, the point of view which we would like to emphasize is the similarity of these generalizations of gauge theories to a spontaneously broken Yang-Mills gauge theory. We begin this section with a discussion of vortices in Higgs theories.

In the simplest case which we discuss first, the Higgs fields are sufficiently numerous to transform effectively (with trivial little group) under $G / C$ where $C$ is the center of $G$. In the region where the Higgs potential is a minimum, $G / C$ is completely broken. Below we will see that in this phase we have the term $\left(f^{2} / 2\right) \operatorname{Tr} P^{2}$ in the Lagrangian, and the Lagrangian describes Goldstone modes. However, there may be regions where the Higgs field is zero. It will be shown that these regions can then act as sources of "vorticity," and in their interior the term $\mathrm{Tr}^{2}$ is absent in the effective Lagrangian. Consequently, the full gauge symmetry is restored in the regions of vanishing Higgs field.

In general the Higgs field can of course break the symmetry in a complicated pattern depending on the little group of its vacuum value. Then we find the more general Lagrangian $(3.26)$ in the Higgs phase. Whether or not topological vortices are possible now depends on the properties of the Higgs minimum under the action of $G$.

To begin with we assume that the Higgs field is a real $[G] \times[G]$ matrix

$$
\Phi=\left[\Phi_{a_{b}}\right], a, b=1,2, \ldots,[G]
$$

with the response

$$
\Phi \rightarrow \operatorname{Ad} s \Phi \mathrm{Ad}^{-1}
$$

under local gauge transformations $s(x) \in G$. Here, $\{$ Ads $\}$ is the adjoint representation AdG of $G$. The Higgs potential is assumed to be such that at its minimum

$$
\Phi=f R
$$

for some $R(x) \in \operatorname{AdG}$. Here $f$ is a constant. In the vacuum sector $\Phi$ has the form (4.3) for all $x$.

Consider now the Lagrangian

$$
\begin{aligned}
\mathscr{L}= & -\frac{f}{4} \epsilon^{\mu \nu \lambda \rho} \operatorname{Tr} F_{\mu \nu}(V) \alpha_{\lambda \rho} \\
& -\frac{1}{2} \operatorname{Tr}\left(D_{\mu}(V) \Phi\right)^{\dagger}\left(D^{\mu}(V) \Phi\right)-V(\Phi),
\end{aligned}
$$

where $V_{\mu}$ is the Yang-Mills potential 


$$
V_{\mu}=i V_{\mu}^{\alpha} L(\alpha),
$$

$f$ is a constant, $V(\Phi)$ is the Higgs potential, and

$$
D_{\mu}(V) \Phi=\partial_{\mu} \Phi+\left[\operatorname{ad} V_{\mu}, \Phi\right] \text {. }
$$

[Here $\operatorname{ad} V_{\mu}=V_{\mu}^{\alpha} \operatorname{ad} L(\alpha)$, where $\operatorname{ad} L(\alpha)$ represents $L(\alpha)$ in the adjoint representation $\mathrm{adG}$ of the Lie algebra $G$.] This $\mathscr{L}$ is invariant under the gauge transformations

$$
\begin{aligned}
& V_{\mu} \rightarrow s V_{\mu} S^{-1}+s \partial_{\mu} s^{-1}, \\
& \Phi \rightarrow \operatorname{Ad} s \Phi \operatorname{Ad} s^{-1}, \\
& \alpha_{\mu \nu} \rightarrow s \alpha_{\mu \nu} S^{-1} .
\end{aligned}
$$

In the vacuum sector we can substitute $(4.3)$ in $\mathcal{L}$. Redefining

$$
\begin{aligned}
& P_{\mu}=R^{-1} V_{\mu} R+R^{-1} \partial_{\mu} R, \\
& A_{\mu \nu}=R^{-1} Q_{\mu_{\nu}} R
\end{aligned}
$$

(4.4) becomes

$$
-\frac{f}{4} \epsilon^{\mu \nu \lambda \rho} \operatorname{Tr} F_{\mu \nu}(P) A_{\lambda \rho}+\frac{f^{2}}{2} \operatorname{Tr} P^{2}
$$

which is identical to (3.6).

Consider now an excited configuration of the Higgs field at a given time, where $f$, instead of being a constant, is a function $\lambda(x)$. Suppose also that the following is true: (a) $\lambda(x)$ vanishes on a string $S$; (b) as $x$ traces a path $\odot_{0}$ encircling $S$, $R(x)$ traces a path in $G / C$ which is not homotopic to a point.

Such a field $\Phi$ is not singular. For although $R$ is singular on $S, \Phi$ vanishes on $S$. We can in fact arrange for $\Phi$ to be quite smooth on $S$ by a suitable choice of $\lambda$. The energy associated with such a $\Phi$ is thus finite.

Far from $S$, finiteness of energy requires that $\lambda \rightarrow f$. For such a $\Phi$, far from $S$, $\&$ still formally reduces to (4.9) after the gauge transformations (4.8). We want, however, to exhibit the new topology of $P_{\mu}$ induced by that of $R$. This is accomplished if we recall from previous discussion ${ }^{16}$ that

$$
f F_{\mu \nu}(P)=-\frac{i}{2} \epsilon_{\mu \nu \lambda \rho} \int_{E} d^{2} \sigma \delta^{4}(x-z(\sigma)) \sigma^{\rho \lambda}(\sigma) .
$$

As we saw, this equation can be reproduced by adding the term

$$
\frac{i}{2} \int_{E} d^{2} \sigma \delta^{4}(x-z(\sigma)) \operatorname{Tr}\left[A_{\mu \nu} \sigma^{\mu \nu}-J^{a b}\left(D_{a} \chi_{b}-D_{b} \chi_{a}\right)\right]
$$

to the action.

We now consider a more general pattern of symmetry breaking by the Higgs field. Let $\Phi$ $=\left\{\phi_{1}, \phi_{2}, \ldots\right\}$ transform as some representation $\Gamma=\{D(g)\}$ of $G$. Let the Higgs minima be located at the configurations

$$
\phi_{i}=D_{i j}(\bar{g}) f_{j},
$$

where the stability group of the constant vector $f$ is $H=\{h\}$ [with representatives $\{D(h)\}$ in $\Gamma$ ]:

$$
D(h)_{i j} f_{j}=f_{i} \text {. }
$$

[The elements $\bar{g} \in G$ label the cosets $G / H$. See after (3.23).] Then the mass term of vector fields is

$$
\frac{1}{2} M_{i j} \hat{P}_{\mu}^{i} \hat{P}^{\mu j} .
$$

Here $P_{\mu}$ is the gauge transform of $V_{\mu}$ by $\bar{g}$, and $\hat{P}_{\mu}$ is related to $P_{\mu}$ as in (3.19). The indices $i, j$ are associated with the $S(i)$ [cf. (3.15)]. The matrix $M$ is given by

$$
M_{i j}=f^{\dagger} S(i) S(j) f
$$

It is invariant under $D(h)$ :

$$
D(h) M \tilde{D}(h)=M .
$$

Thus the model we get is the generalized nonlinear model of $(3.26)$.

In this model as well there can be vortices. For instance, let the space of the Higgs minima be multiply connected. Let $S$ be a string of zeros of $\Phi$. Let $\rho_{0}$ be a path at a given time encircling $S$ on which $\Phi$ has the form (4.10). Then if $\Phi$ traces a path which is not homotopic to the identity as $\chi$ traces $\rho_{0}$, the gauge transformation from $V$ to $P$ induces a vortex in $P$.

\section{THE 't HOOFT ALGEBRA}

In his work on the phases of gauge theories, 't Hooft ${ }^{17}$ introduced the algebra involving a pair of operators $A$ and $B$. In this section we indicate how this algebra can be realized using the new gauge fields.

Ostensibly the $A$ and $B$ operators made from these generalized gauge fields are not similar to those in Yang-Mills theories. The types of gauge transformations which act on these fields are different. However, in view of the remarks of the previous section, perhaps the fact that this algebra as well can be constructed in the generalized gauge theories shows that there is indeed a close relation between the two gauge theories.

In any domain $D$ (at a given time) which is not intersected by the string, $F_{\mu \nu}(P)=0$ by (3.7). Thus $P$ is locally a pure gauge:

$$
\begin{aligned}
& P_{\mu}=g^{-1} \partial_{\mu} g, \text { in } D, \\
& g(x) \in G .
\end{aligned}
$$

The group $G$ acts by conjugation on $P_{\mu}, \omega$, and $A_{\mu \nu}$ : 


$$
\begin{aligned}
& P_{\mu} \rightarrow s P_{\mu} S^{-1}, \\
& \omega \rightarrow S \omega S^{-1}, \\
& A_{\mu \nu} \rightarrow S A_{\mu \nu} s^{-1}, \quad s \in G .
\end{aligned}
$$

Thus the center $C$ of $G$ acts trivially on the $P$ and $\omega$. The effective group is $G / C$, that is, the adjoint group AdG. Thus with no loss of content, we can write

$$
\operatorname{ad} P_{\mu}=[\operatorname{Adg}]^{-1} \partial_{\mu}[\mathrm{Adg}] \text {. }
$$

We recall that $[\mathrm{Ad} g]$ is the adjoint representation $\operatorname{Ad} G$ of $G$ and $\operatorname{ad} G$ is the Lie algebra of the representation $\mathrm{Ad} G$.

If $C$ is nontrivial, then certainly $\mathrm{Ad} G$ is multiply connected. In general if $D$ is multiply connected, Ad $g$ is not globally defined by ad $P .{ }^{18}$ However, we will assume the analog of the flux quantization condition in type-II superconductors. The $\mathrm{Adg}$ is globally defined.

In the presence of a string, the space $D$ is in general multiply connected. For instance, let $S$ be the (closed or infinite) string and $D=\mathrm{R}^{3} / S$. Then a path $\odot_{0}$ which encircles $S$ cannot be shrunk to a point. Such a $D$ is multiply connected. The path $\operatorname{Adg}\left(\boldsymbol{\odot}_{0}\right)$ in $\operatorname{AdG}$ associated with such a $\boldsymbol{P}_{0}$ need not be homotopic to a point.

We can integrate $(5.3)$ in $D$ :

$$
\operatorname{Adg}(x)=O^{*} \exp \left(\int_{x_{0}}^{x} \operatorname{ad} P_{\mu} d x^{\mu}\right) \operatorname{Ad} g\left(x_{0}\right) .
$$

The integration is along any path in $D$ from $x_{0}$ to $x$ and $O^{*}$ denotes antipath ordering. For a path $\odot$ homotopic to a point, as $x$ traces $P$,

$$
\operatorname{Adg}(x) \operatorname{Adg}\left(x_{0}\right)^{-1}=O^{*} \exp \left(\int_{x_{0}}^{x} \operatorname{ad} P_{\mu} d x^{\mu}\right)
$$

traces a path in $\mathrm{Ad} G$ homotopic to a point. For a path such as $\odot_{0}$, this need not be the case.

Suppose it is not the case. As we shrink $P_{0}$ towards the string $S$, the path in $\operatorname{Ad} G$ changes continuously. If $\operatorname{ad} P$ were well defined also on $S$, this would mean that the path in $\mathrm{AdG}$ can be continuously shrunk to a point contrary to hypothesis. Thus ad $P$ is singular on $S$.

It is easy to specify the nature of this singularity. For $\rho_{0}$ very near $S,{ }^{19}$

$$
O^{*} \exp \left(\oint_{x_{0}} \operatorname{ad} P_{\mu} d x^{\mu}\right)=1+\int_{\Sigma} \operatorname{ad} F_{\mu \nu}(P) d x^{\mu} \wedge d x^{\nu}
$$

The symbol $\oint_{x_{0}}$ indicates integration on a small circle $\rho_{0}$ starting at $x_{0}$. It bounds the surface $\sum$ (which in turn is intersected by $S$ ). Thus the singularity is in $\operatorname{ad} F(P)$. It is a (non-Abelian) vortex in $P^{16}$ :

$$
\operatorname{ad} F_{\mu \nu}(P)=-\frac{i}{2} \epsilon_{\mu \nu \rho \sigma} \int d^{2} \sigma \delta^{4}(x-z(\sigma)) \epsilon^{a b} \partial_{a} z^{\rho} \partial_{b} z^{\sigma} \operatorname{ad} \omega
$$

Here by $(5.6)$ we find

$$
\begin{aligned}
1+\int \operatorname{ad} F_{\mu \nu} d x^{\mu} \quad d x^{\nu} & \cong \exp \left(\int \operatorname{ad} F_{\mu \nu} d x^{\mu} d x^{\nu}\right) \\
& =\exp (-i \operatorname{ad} \omega)=1
\end{aligned}
$$

since $\operatorname{Ad} g(x) \operatorname{Ad} g\left(x_{0}\right)^{-1}$ approaches identity as $x$ completes one cycle in $P_{0}$. Equation (5.8) is the generalization of the familiar flux quantization condition $(\omega$ in this section differs from those in the earlier ones by a factor $1 / f$ ).

If these equations are written in the defining representation and not in the adjoint representation, the unity in (5.8) is replaced by a suitable central element $z$ of $G$ :

$$
O^{*} \exp \left(\oint_{x_{0}} P_{\mu} d x^{\mu}\right)=z
$$

Consider now the second-quantized version of this theory. Let $A\left(\odot, x_{0}\right)$ be the operator

$$
A\left(\boldsymbol{\rho}, x_{0}\right) \equiv \frac{1}{N} \operatorname{Tr} O^{*} \exp \left(\oint_{x_{0}} P_{\mu} d x^{\mu}\right) .
$$

Here $\rho$ is a closed path from $x_{0}$ to $x_{0}$, the integral is evaluated over $\rho$, and $N$ is the dimension of the representation $\{L(\alpha)\}$. Let $B(S)$ be the creation operator for a string $S$ (at a given time, so $S$ is the time slice of the string). Then the previous analys is shows that if $\odot$ does not link $S$,

$$
A\left(\odot, x_{0}\right) B(S)=B(S) A\left(\odot, x_{0}\right) .
$$

But if $\odot$ is a path $\odot_{0}$ which links $S$ once,

$$
A\left(\odot, x_{0}\right) B(S)=z B(S) A\left(\odot, x_{0}\right) .
$$

The algebra defined by $(5.11)$ and $(5.12)$ is due to 't Hooft.

\section{GENERALIZED GAUGE FIELDS AND DUAL DESCRIPTIONS OF PHONONS}

In this section, we discuss the new gauge fields with regard to the notion of duality. As we have seen in Secs. II and III the potentials $A_{\mu \nu}$ give a representation of field theories of spin-0 excitations. These scalar excitations or "phonons" can be regarded as the description of one phase of a larger system. Just as electromagnetism admits a dual description either in terms of globally defined electric potentials and electric sources or globally defined magnetic potentials and magnetic sources, it will now be shown that an analogous situation holds for the new gauge systems. The non-Abelian system requires that some restriction be placed on the possible homogeneous spaces $G / H$ 
if the duality is to be valid. We find that $G / H$ must by a symmetric space.

In the Ginzburg-Landau theory, the phase $\theta$ of the complex order parameter $\Phi$ describes the phonon mode. Here

$$
\Phi=e^{i \theta} \lambda, \lambda \text { real. }
$$

In analogy, for the non-Abelian case, we can say the following: let $\Phi=\left\{\Phi_{1}, \Phi_{2}, \ldots\right\}$ transform under the representation $\Gamma=\{D(g)\}$ of $G$. Following $\mathrm{Kibble}^{20}$ we can write its polar decomposition

$$
\phi_{i}=D(\bar{g})_{i j} \lambda_{j}, \quad \bar{g} \in G .
$$

We call the degrees of freedom associated with $\vec{g}$ phonon modes. (Recall that $\bar{g}$ labels $G / H$ where $H$ is the stability group of the vector $\lambda$.)

In electromagnetism, in the normal description, globally defined potentials are introduced for the field $F_{\mu \nu}$ :

$$
F_{\mu \nu}=\partial_{\mu} A_{\nu}-\partial_{\nu} A_{\mu} .
$$

As a consequence, there can be no magnetic sources for $F_{\mu \nu}$ :

$$
\partial_{\mu}^{*} F^{\mu \nu}=0 \text {. }
$$

There can of course be electric sources $J_{\mu}$ :

$$
\partial_{\mu} F^{\mu \nu}=J^{\nu} .
$$

In the dual description, potentials are introduced for $* F^{\mu \nu}$ :

$$
* F_{\mu \nu}=\partial_{\mu} B_{\nu}-\partial_{\nu} B_{\mu} .
$$

This allows magnetic, but not electric sources:

$$
\partial_{\mu} F^{\mu \nu}=0, \partial_{\mu}{ }^{*} F^{\mu \nu}=K^{\nu} .
$$

The potentials $A_{\mu}$ and $B_{\mu}$ provide dual descriptions of electromagnetism. We call the electric and magnetic charges which create the currents $J_{\mu}$ and $K_{\mu}$ dual sources. If both are present and are point particles, the equations

$$
\partial_{\mu} F^{\mu \nu}=0, \quad \partial_{\mu}^{*} F^{\mu \nu}=0
$$

are valid away from the charges. We know that as a consequence either of the potentials $A_{\mu}$ or $B_{\mu}$ exists locally but not globally. Further, the topological and angular momentum properties of the system are profoundly affected.

Let us now discuss analogous dual possibilities for the phonon modes. We first examine the Abelian case. For this case, the ideas below are in the paper of Rasetti and Regge. ${ }^{2}$

\section{The Abelian case}

The role of $F_{\mu \nu}$ is now played by the current $P_{\mu}$ associated with the global phase change

$$
\Phi \rightarrow e^{i \alpha} \Phi, \quad \alpha=\text { constant. }
$$

In the phase where $\lambda$ is real and only phonon excitations are present, we see from (6.1) that this current is, up to a constant,

$$
P_{\mu}=\partial_{\mu} \theta
$$

[for simple Lagrangians like $-\left|\partial_{\mu} \Phi\right|^{2}-V(\Phi)$ ]. Because of this form, $P_{\mu}$ is curl free:

$$
\partial_{\mu} P_{\nu}-\partial_{\nu} P_{\mu}=0 \text {. }
$$

This equation is the analog now of (6.4). Since the phonon mode can couple to sources, we also have the analog of (6.5):

$$
\partial_{\mu} P^{\mu}=\rho,
$$

where $\rho$ is not necessarily zero.

Thus $\theta$ is similar to $A_{\mu}$. It is the potential which solves the equation (6.11). In the dual approach, we attempt to construct a potential for

$$
\partial_{\mu} P^{\mu}=0 \text {. }
$$

Such a potential is just the antisymmetric field $A_{\mu \nu}$ :

$$
P^{\mu}=\frac{1}{2 f} \epsilon^{\mu \alpha \beta \gamma} \partial_{\alpha} A_{\beta \gamma} .
$$

For this system, we have seen that $P_{\mu}$ need not be irrotational (Sec. II):

$$
\partial_{\mu} P_{\nu}-\partial_{\nu} P^{\mu}=S_{\mu \nu} \neq 0 .
$$

For the field $P_{\mu}, \theta$ and $A_{\mu \nu}$ are thus dual potentials. We now turn to the dual sources. For brevity, let us introduce differential forms

$$
\begin{aligned}
& P=P_{\mu} d x^{\mu}, \\
& F=d P=\frac{1}{2}\left(\partial_{\mu} P_{\nu}-\partial_{\nu} P_{\mu}\right) d x^{\mu} \wedge d x^{\nu}, \\
& * P=\frac{1}{3 !} \epsilon_{\mu \nu \lambda \rho} P^{\mu} d x^{\nu} \wedge d x^{\lambda} \wedge d x^{\rho}, \\
& d^{*} P=\frac{1}{4 !} \partial_{\lambda} P^{\lambda} \epsilon_{\mu \nu \rho \sigma} d x^{\mu} \wedge d x^{\nu} \wedge d x^{\rho} \wedge d x^{\sigma} \\
& \quad \equiv \partial_{\lambda} P^{\lambda} d V .
\end{aligned}
$$

Equation (6.12) is thus

$$
d^{*} P=\rho d V
$$

and Eq. (6.15) is

$$
d P=S=\frac{1}{2} S_{\mu \nu} d x^{\mu} \wedge d x^{\nu} .
$$

We require the elementary dual sources to have the following properties: (1) They vanish outside a submanifold of dimension $<4$ in Minkowski space $\mathfrak{m}^{4}$; this requirement is suggested by the fact that electric and magnetic charges form lines of dimension 1 in Minkowski space; (2) their presence should induce a nontrivial topology (cohomology) either in ${ }^{*} P$ or $P$. Thus, suppose $\rho$ has support in $K$. Then 


$$
d^{*} P=0 \text { in } \mathfrak{M}^{4} / K
$$

We require then that the closed three-form $* P$ is not exact in $\mathfrak{N}^{4} / K$; that is, there does not exist a global potential $A_{\lambda \rho}$ in $\mathfrak{m}^{4} / K$ such that $(6.14)$ is valid; similarly, if $\bar{K}$ is the support of $S$, we require that there does not exist a globally defined potential (zero) form $\theta$ in $\mathfrak{M}^{4} / \bar{K}$ such that $(6.10)$ is valid. These requirements are in analogy to electromagnetism; they determine the nature of the sources.

Consider the source $S$ first. In $\mathfrak{M}^{4} / \bar{K}, P$ is a closed one-form. If $\mathfrak{T}^{4} / \bar{K}$ is simply connected, then by a well-known result, ${ }^{21} P$ is exact. Thus $\mathfrak{T}^{4} / \bar{K}$ must be multiply connected. We can achieve this by assuming that $\bar{K}$ is a (closed or infinitely long) string. Thus, an elementary source for $\left(6.15^{\prime}\right)$ is a string. The form of $S$ in this case was discussed previously.

As regards $\rho$, note that $* p$ is a three-form. A simple way to have closed but not exact $* P$ in $\mathbb{N}^{4} K$ is to assume that

$$
\mathfrak{T}^{4} / K=S^{3} \times \mathrm{R}^{1} .
$$

For there exist closed three-forms such that

$$
\int_{S^{3}} * P \neq 0
$$

This means, by Stokes's theorem, that $A_{\lambda \rho}$ does not exist. (An example of such a $* P$ is the standard volume form on $S^{3}$.) Now the topology $S^{3} \times R^{1}$ can be achieved if $\rho$ is given by

$$
\rho(x)=\rho_{0} \delta^{4}\left(x-x_{0}\right), \quad x_{0} \text { constant. }
$$

In this case, the radial coordinate $r$ (centered at $x_{0}$ fulfills the identity

$$
r>0
$$

in $\mathfrak{T}^{4} / K$ and spans an $\mathrm{R}^{1}$. The angular coordinates $\operatorname{span} S^{3}$.

We note that with such sources, $P$ and $* P$ in fact fail to be exact. For $P$, we saw this earlier: let $\odot_{0}$ be a closed curve encircling the string. Then since

$$
\int_{\mathcal{P}_{0}} P \propto \omega \neq 0
$$

$P$ cannot have the form (6.10) globally. A similar argument works for $* P$ since

$$
\int_{S^{3}} * P \propto \rho_{0} \neq 0 \text {. }
$$

Here $S^{3}$ is any three-sphere encircling $x_{0}$.

We have already interpreted the string as a vortex line in the field $P_{\mu}$. It remains to interpret $\rho$. For this we can follow Rasetti and Regge. ${ }^{2}$ Note that $(6.12)$ has the solution

$$
\begin{aligned}
& P_{\mu}=-\rho_{0} \delta^{(3)}\left(x-x_{0}\right) \theta\left(t-t_{0}\right) n_{\mu}+\bar{P}_{\mu}, \\
& n_{\mu}=(0,0,0,1),
\end{aligned}
$$

where $\bar{P}_{\mu}$ is a solution of the homogeneous equation. Thus the density $\rho_{0}$ is augmented at $x=x_{0}$, perhaps by an external agency. If $P_{\mu}$ can be suitably interpreted as the mass current of superfluid helium, the source $\rho$ describes the injection of helium atoms into the system.

Some such interpretation is suggested by the work of Rasetti and Regge. In the nonrelativistic analog of this system, they have interpreted $\rho$ in the preceding fashion and derived the FeynmanOnsater relation. The latter is the analog of the Dirac quantization condition on the product of electric and magnetic charges.

The implications of the existence of dual sources for $P_{\mu}$ (even in the Abelian case) have not been systematically investigated in the literature. The work of Rasetti and Regge ${ }^{2}$ is an exception.

\section{The non-Abelian case}

The existence of dual sources can be shown for the non-Abelian case as well when $G / H$ is a symmetric space.

The role of the field strength is played now by the current $I_{\mu}$ associated with the internal symmetry of the theory. In the Abelian case $I_{\mu}=P_{\mu}$; but in the non-Abelian case [cf. (3.20)], they differ from each other. The description of the theory using the group element $g$ corresponds to the description which uses $\theta$ in the preceding subsection. The dual description uses the non-Abelian $A_{\mu \nu}$.

We first consider the description which uses $g$. The Lagrangian (3.20) is invariant under the global transformation

$$
\delta g=i \epsilon_{\alpha} L(\alpha) g
$$

( $\epsilon_{\alpha}$ constant). The associated conserved current is $I_{\mu}^{\alpha}$ where (up to a constant)

$$
I_{\mu}^{\alpha} L(\alpha)=I_{\mu}=-g S(i) g^{-1} \operatorname{Tr} S(i) g^{-1} \partial_{\mu} g=-g \hat{P}_{\mu} g^{-1} .
$$

If we can show that this can be written in the form

$$
\begin{aligned}
& I_{\mu}=c U^{-1} \partial_{\mu} U, \\
& U \in G, \quad c=\text { constant },
\end{aligned}
$$

then

$$
F_{\mu \nu}(I / c)=0
$$

and we have the analog of the Abelian equation (6.11).

To prove this, we require the additional assumption 


$$
[S(i), S(j)] \in \underline{H} .
$$

(In such cases, $G / H$ is said to be symmetric.) We may also note the commutation relations

$$
\begin{aligned}
& {[T(\alpha), T(\beta)] \in \underline{H},} \\
& {[T(\alpha), S(i)]=\bar{d}_{\alpha i j} S(j),}
\end{aligned}
$$

which are always valid [the last follows from (3.23)]. Such an algebra allows the automorphism $\tau$ where

$$
\begin{aligned}
& T(\alpha)_{\vec{\tau}} T_{\tau}(\alpha)=T(\alpha), \\
& S(i)_{\vec{\tau}} S_{\tau}(i)=-S(i) .
\end{aligned}
$$

(Elsewhere ${ }^{12}$ it is shown that many physically interesting models allow the automorphism $\tau$.) Let us use the same symbol $\tau$ to denote the induced automorphism on the group:

$$
\begin{aligned}
g & =\exp \left\{i\left[\xi_{2} T(\alpha)+\eta_{i} S(i)\right]\right\} \\
& \vec{\tau} g_{\tau}=\exp \left\{i\left[\xi_{\alpha} T(\alpha)-\eta_{i} S(i)\right]\right\} .
\end{aligned}
$$

Now if

$$
g^{-1} \partial_{\mu} g=T(\alpha) \operatorname{Tr}\left[T(\alpha) g^{-1} \partial_{\mu} g\right]+S(i) \operatorname{Tr}\left[S(i) g^{-1} \partial_{\mu} g\right],
$$

then

$$
\begin{aligned}
g_{\tau}^{-1} \partial_{\mu} g_{\tau}= & T(\alpha) \operatorname{Tr} T(\alpha) g^{-1} \partial_{\mu} g \\
& -S(i) \operatorname{Tr}\left[S(i) g^{-1} \partial_{\mu} g\right]
\end{aligned}
$$

and

$$
\begin{gathered}
I_{\mu}=\frac{1}{2} U^{-1} \partial_{\mu} U, \\
U=g_{\tau} g^{-1} .
\end{gathered}
$$

We have thus shown (6.27). In this description, in the presence of external sources, there is no reason for $\partial_{\mu} I^{\mu}$ to vanish. But $F_{\mu \nu}(2 I)$ is zero so long as it is globally defined.

For the dual description, we go to the Lagrangian (3.17) with the interaction (3.28). The representation of $P_{\mu}$ in that Lagrangian allows for vortices in $P_{\mu}$ [Eq. (3.33)] while $\partial_{\mu} P^{\mu}=0$ [Eq. $(3.35)]$.

\section{ACKNOWLEDGMENT}

A.P.B., V.P.N., and C.G.T. acknowledge support from the U. S. Department of Energy.
*Address after 1 April 1981: Institute of Theoretical Physics, FACK, S-402 20 Göteborg 5, Sweden.

${ }^{1}$ V. I. Ogievetsky and I. V. Polubarinov, Yad. Fiz. $\underline{4}$, 216 (1966) [Sov. J. Nucl. Phys. 4, 156 (1967)]; S. Deser, Phys. Rev. 187, 1931 (1969); K. Hayashi, Phys. Lett. 44B, 497 (1973); M. Kalb and P. Ramond, Phys. Rev. $\overline{\mathrm{D}} \underline{9}, 2273$ (1974); E. Cremmer and J. Scherk, Nucl. Phys. B72, 117 (1974); Y. Nambu, Phys. Rep. 23C, 250 (1976); in Quark Confinement and Field Theory, edited by D. R. Stump and D. H. Weingarten (Wiley, New York, 1977); A. Sugamoto, Phys. Rev. D 19, 1820 (1979); K. Seo, M. Okawa, and A. Sugamoto, ibid. 19 , 3744 (1979).

${ }^{2}$ F. Lund and T. Regge, Phys. Rev. D 14, 1524 (1976). See also M. Rasetti and T. Regge, Physica (Utrecht) 80A, 217 (1975).

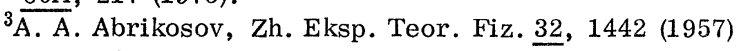
[Sov. Phys.-JETP $\underline{5}, 1174$ (1957)].

${ }^{4}$ H. B. Nielsen and P. Olesen, Nucl. Phys. B61, 45 (1973).

${ }^{5} \mathrm{Cf}$. P. K. Townsend, in Supergravity, proceedings of the Stony Brook Supergravity Workshop, edited by P. van Nieuwenhuizen and D. Z. Freedman (North-Holland, Amsterdam, 1979); D. Z. Freedman and P. K. Townsend, Nucl. Phys. B1.77, 282 (1981).

${ }^{6}$ A. P. Balachandran, G. Marmo, B. S. Skagerstam, and A. Stern, J. Phys. G 7, 1001 (1981).

${ }^{7}$ A. Chodos, R. L. Jaffe, K. Johnson, C. B. Thorn, and V. F. Weisskopf, Phys. Rev. D 9, 3471 (1974).

${ }^{8}$ See C. Rebbi, Phys. Rep. 12C, 1 (1974); J. Scherk,
Rev. Mod. Phys. 47, 123 (1975).

${ }^{9}$ G. 't Hooft, Nucl. Phys. B138, 1 (1978); B153, 141 (1979).

${ }^{10}$ See M. Rasetti and T. Regge, Ref. 2.

${ }^{11}$ See A. P. Balachandran, G. Marmo, B. S. Skagerstam, and A. Stern, Nucl. Phys. B162, 385 (1980).

${ }^{12}$ A. P. Balachandran, R. Ramachandran, H. Rupertsberger, and B. S. Skagerstam, Lett. Math. Phys. $\underline{4}$, $79(1980)$ and references therein; E. Brezin, S. Hikami, and J. Zinn-Justin, Nucl. Phys. B165, 528 (1980); H. Eichenherr and M. Forger, ibid. B155, 381 (1979).

${ }^{13}$ A. P. Balachandran, B. S. Skagerstam, and A. Stern, Phys. Rev. D 20, 439 (1979).

${ }^{14}$ Y. Ne'eman, Texas report, 1980 (unpublished).

${ }^{15}$ A. P. Balachandran, A. Stern, and G. Trahern, Phys. Rev. D 19, 2416 (1979) and references therein.

${ }^{16}$ F. Englert and P. Windey, Nucl. Phys. B135, 529 (1978); Phys. Rep. 49C, 173 (1979).

${ }^{17} \mathrm{G}$. 't Hooft, Lectures at the 1980 Schladming Winter School (unpublished), and Ref. 9.

${ }^{18} \mathrm{~A}$. P. Balachandran and G. Marmo, Lett. Nuovo Cimento 27, 13 (1980); J. Kiskis, Phys. Rev. D 15, 2329 (1977); 16,2535 (1977).

${ }^{19}$ I. Ya. Aref'eva, Phys. Lett. 95B, 269 (1980); N. E. Bralic, Phys. Rev. D 22, $30 \overline{90}(1980)$; P. M. Fishbane, S. Gasiorowicz, and P. Knaus, ibid. 24, 2324 (1981); M. B. Mensky, Lett. Math. Phys. $\underline{3}, \overline{513}$ (1979).

${ }^{20}$ T. W. B. Kibble, Phys. Rev. 155, 1554 (1967).

${ }^{21} \mathrm{H}$. Flanders, Differential Forms (Academic, New York, 1963). 